\title{
Role of mature sphingolipids in yeast: new tools
}

\author{
Andreas Conzelmann* \\ Department of Biology, University of Fribourg, Fribourg, \\ Switzerland.
}

\section{Summary}

Sphingolipids of yeast have been described as being important for numerous cell biological phenomena such as heat resistance, endocytosis, stress resistance and many others. The genetic or pharmacological elimination of specific features or entire classes of sphingolipids has pinpointed specific sphingolipids as pivotal regulators in many processes. The report by Epstein et al. adds two new tools for such studies: a strain being completely resistant to aureobasidin A, a specific inhibitor of inositol phosphorylceramide synthase and a second strain where this synthase is deleted. The resulting phenotypes advocate new roles of complex sphingolipids in cytokinesis, lipid droplet biogenesis and cell survival.

\section{Introduction}

Most genes for sphingolipid biosynthesis and degradation in yeast have been identified (Fig. 1) and there now is a wealth of data demonstrating that sphingolipids are required for numerous essential and non-essential cell biological phenomena such as resistance to heat, high salt, low $\mathrm{pH}$, endocytosis, vacuolar acidification, cell wall integrity, chronological aging, transport of GPI proteins and nutrient transporters from the ER to the plasma membrane, functionality of plasma membrane nutrient transporters, and many others (for review see Dickson, 2010). In only very few cases, however, a given sphingolipid has been shown to bind directly to a particular target protein in the same way as for instance C18-sphingomyelin has recently been shown to bind to the transmembrane domain of mammalian p24 (Contreras et al., 2012). In a majority of studies, specific sphingolipids have been proposed to be required for a specific process mainly through the comparison of phenotypic consequences of successive gene dele-

${ }^{*}$ For correspondence. E-mail andreas. conzelmann@unifr.ch; Tel. (+41) 2630086 31; Fax (+41) 2630097 35. tions along the biosynthetic pathway. For example, if a given process $\mathrm{X}$ is not affected by deletion of IPT1 but by deletion of CSG2 (Fig. 1), one may conclude that the process depends on mannosyl-inositol phosphorylceramide (MIPC). Yet, it also needs to be considered that the process $\mathrm{X}$ may be perturbed by accumulation of inositol phosphorylceramide (IPC), a possibility that can be excluded if process $X$ is also blocked by aureobasidin $A$ $(\mathrm{AbA})$, a highly specific pharmacological inhibitor of IPC synthase Aur1p. Indeed, several other mutations have been reported where the accumulation of upstream rather than the lack of downstream metabolites seems to be responsible for a compromised process X (Saba et al., 1997; Beeler et al., 1998; Schorling et al., 2001; Aguilar et al., 2010). Thus, downregulation of many successive enzymatic steps is required to pinpoint the specific lipids required for a specific cellular function.

\section{Two new tools}

In this issue, two new tools for yeast sphingolipid research are introduced by the paper by Epstein et al. The report introduces a cell line, which is completely $\mathrm{AbA}$ resistant even though it makes high amounts of ceramides. It furthermore relates the successful deletion of $A U R 1$ in this strain, thereby for the first time creating yeast cells that lack all complex sphingolipids (Fig. 1). Aur1p transfers inositolphosphate from phosphatidylinositol (PI) onto ceramides to form IPC at the luminal side of Golgi membranes (Nagiec et al., 1997; Levine et al., 2000). Similar to ergosterol biosynthesis, the early steps of yeast sphingolipid biosynthesis are covered by essential genes or redundant gene pairs, simultaneous deletion of which is lethal (red and green, respectively, in Fig. 1). A second site suppressor mutation can rescue $\mathrm{lcb} 1 \Delta$ cells, and the lag $1 \Delta \operatorname{lac} 1 \Delta$ double deletion is not lethal in all genetic backgrounds, but no suppressors were found so far for deletions abolishing fatty acid elongation or IPC synthesis. Also, AbA has been reported to rapidly kill cells (Endo et al., 1997). Similarly, the biosynthesis of sphingomyelin, the mammalian counterpart of IPCs, is essential for proliferation of mammalian cells (Tafesse et al., 2007). A first hint that lethality of the $A U R 1$ deletion may be rescued came from the observation that a lag $1 \Delta \operatorname{lac} 1 \Delta \mathrm{ypc} 1 \Delta \mathrm{ydc} 1 \Delta(4 \Delta)$ mutant, largely unable 


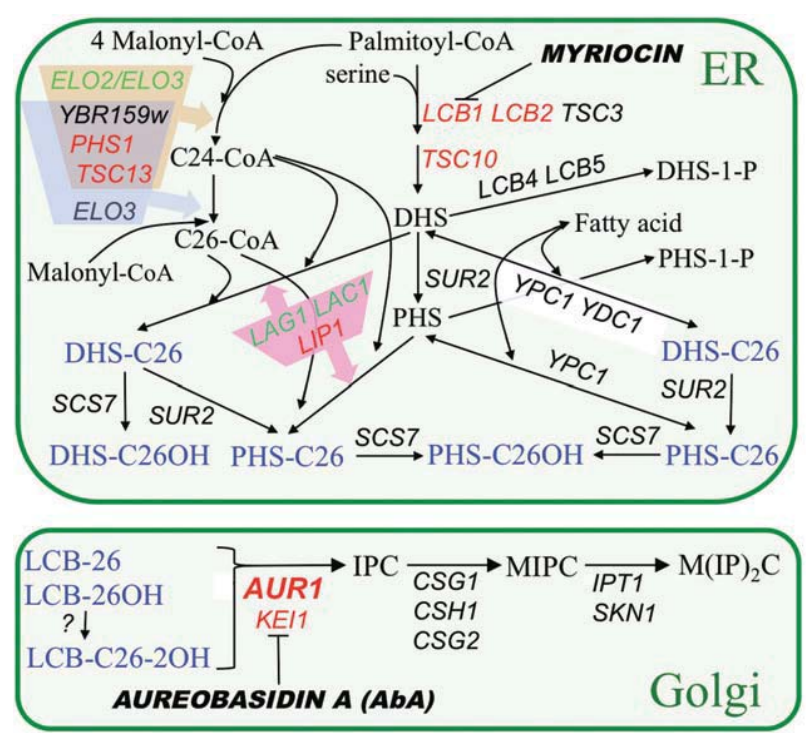

Fig. 1. Major pathways of sphingolipid biosynthesis in yeast. Gene names are in italic and inhibitors in bold italics. DHS, dihydrosphingosine; IPC, inositol phosphorylceramide; LCB, long chain base; MIPC, mannosyl-IPC; P, phosphate; PHS, phytosphingosine; $\mathrm{M}(\mathrm{IP})_{2} \mathrm{C}$, inositolphosphoryl-MIPC. Essential genes in red, redundant gene pairs, simultaneous deletion of which is lethal in green, ceramides in blue.

to make ceramides, is resistant to AbA (Schorling et al., 2001). This observation suggested that aur1 $\Delta$ LAG1 cells may die not for lack of complex sphingolipids, but rather because ceramides reach toxic levels in such cells. The finding of Schorling et al. could not be reproduced by others when very high concentrations of $\mathrm{AbA}\left(2.5 \mu \mathrm{g} \mathrm{ml}^{-1}\right)$ were used to grow the $4 \Delta$ mutant (Cerantola et al., 2009). Building on past experience, Epstein et al. (2012) tried to generate the $A U R 1$ deletion in lag $1 \Delta$ lac $1 \Delta$ cells harbouring GhLAG1, a LAG1 homologue from cotton (Gossypium hirsutum) inserted into the TRP1 locus. The resulting GhLag1 strain (TDH3::GhLAG1::TRP1 lac1 $\Delta$ lag1 $\Delta$ ) differs from normal yeast in two ways: it makes ceramides and complex sphingolipids with $\mathrm{C} 18$ fatty acids rather than C26 fatty acids (Fig. 1) and it contains significantly (threefold) higher amounts of ceramide and also of complex sphingolipids than wild type (WT). This cell line turns out to be completely resistant to AbA although its ceramide levels under AbA still increase by a factor of 3.5 , an increase that is comparable to the one observed for WT cells, whereby WT cells stop growing and die on AbA, whereas GhLag1 continue to grow. The conclusion seems evident: accumulation of C26-ceramides is toxic, accumulation of C18ceramides is not. A recent study demonstrates that elo3 $\Delta$, making C22 and a little C24 but no C26 fatty acids (Fig. 1), are quite AbA-resistant, suggesting also that C22 ceramides are less toxic than C26 ceramides (Tani and Kuge, 2010).
The AbA hyper-resistant phenotype of GhLag1 cells encouraged Epstein et al. (2012) to try to generate a TDH3::GhLAG1::TRP1 lac1 $\Delta$ lag1 $\Delta$ aur1 $\Delta$. Such a strain, called cSL $\Delta$ because lacking all complex sphingolipids, was obtained by sporulating a diploid which is heterozygous at all four loci. This avoids the selection for suppressor mutations and the results very clearly indicate that AUR1 deletion in GhLag1 is possible. ${ }^{1}$

\section{Anything wrong with GhLag1?}

Apart from being AbA resistant, GhLAG1 cells cannot grow in presence of Calcofluor white, YW3548 (an inhibitor of GPI precursor biosynthesis) and rapamycin, indicating some problem in making and remodelling the cell wall. That sphingolipids influence the cell wall architecture had been realized for a long time and by many authors: e.g. lag1 $\Delta$ lac1 $\Delta$ mutants have abnormal, multilayered cell walls and are Calcofluor white hypersensitive (Barz and Walter, 1999). Similarly, growth inhibition by myriocin can be overcome by overexpression of MSS4, ROM2, RHO1 and further downstream elements required for activation of the cell wall integrity pathway (Kobayashi et al., 2005). Part of this rescue may be due to a stimulatory effect of Rom2p on ceramide synthesis indicated by genetic data (Aguilar et al., 2010). One explanation for cell wall abnormalities in sphingolipid-deficient mutants is that numerous cell wall proteins are GPI proteins whose export out of the ER is dependent on ceramide-biosynthesis (Barz and Walter, 1999). However, data of Epstein et al. (2012) suggest that the abundant C18-ceramides and complex C18sphingolipids of GhLag1 are functional for GPI protein transport since, Gas1p, Cwp2p, Pma1p and many other membrane proteins are not accumulating inside GhLag1 cells but are found at the plasma membrane as in WT cells. A similar conclusion was reached in an earlier report where pulse chase studies showed a normal ER to Golgi transport of Gas $1 \mathrm{p}$ in a $4 \Delta$ strain harbouring a murine CERS5, a LAG1 homologue that also makes $\mathrm{C} 16$ and $\mathrm{C} 18$ ceramides. This strain also was Calcofluor white hypersensitive (Cerantola et al., 2007). Therefore, the reason for the cell wall problem of GhLag1 cells is presently unexplained.

\section{What about phenotypes of $c S L \Delta$ ?}

In spite of the robust growth of GhLag1 on $0.5 \mu \mathrm{g} \mathrm{ml}^{-1}$ of $\mathrm{AbA}$, removing their AUR1 leaves the resulting cSL $\triangle$ cells very sick. This is not so surprising since long-term AbA treatment of GhLag1p represses IPC levels without causing a proportional decrease in $\mathrm{M}(\mathrm{IP})_{2} \mathrm{C}$ (Epstein

${ }^{1}$ The lower-than-expected frequency of quadruple mutants (3 instead of the expected 12) is most likely due to the fact that two loci $(A U R 1=$ YKL004w and $L A C 1=$ YKL008c) are linked. 
et al., 2012 herein fig. 2E). It is possible that the partial inhibition of Aur1p creates a situation where the down stream processing steps are no more rate limiting and can handle the entire pool of IPC that still is generated. Epstein et al. (2012) propose that the fragility and slow growth phenotype of cSLC $\Delta$ cells can be explained by two discrete difficulties in cytokinesis. While nuclear division takes place normally, cSL $\Delta$ cells seem to retain the septin protein Cdc10p at the bud neck longer than normal and second, they remain attached to each other while some kind of septum already has created a tight barrier for cytosolic proteins. Importantly, cSL $\Delta$ cells can be grown in liquid culture only when shaking is omitted. This latter phenotype and the ruffled appearance of cSLC $\Delta$ cell walls in scanning electron microscopy suggest that the problem in cell separation and cell wall remodelling is much more severe than in lac1 $\Delta$ lag1 $1 \Delta$ cells alluded to above (Barz and Walter, 1999). This is not astonishing since lac1 $1 \Delta$ lag1 $\Delta$ cells still make small amounts of complex sphingolipids through alternative pathways (Vionnet et al., 2011). The defect of cSL $\Delta$ cells is somewhat reminiscent of the cell separation defect of gpi7 cells making GPI anchors of slightly abnormal structure, a defect that results in the failure to localize the GPI anchored Egt2p $\beta$-glucanase at the bud neck of the daughter cell during abscission (Fujita et al., 2004). Again, the growth defect of gpi7 is much less severe than the one of cSL $\Delta$. A detailed analysis of the events at the bud neck in cSL $\Delta$ cells may therefore bring to light a novel role of some complex sphingolipid in late cytokinesis. More detailed analysis of cSL $\Delta$ cells will also be required to explain the high mortality rate of their daughter cells and their tendency to accumulate lipid droplets.

The cSL $\Delta$ may be deficient in previously reported roles of complex sphingolipids

An early series of studies showed that a lcb1 $1 \Delta$ mutant can live without importing long chain bases (LCBs) from the medium if it can make PI with a C26 fatty acid in the sn-2 position of the L-glycerol moiety due the SLC1-1 gain of function mutation (Nagiec et al., 1993), whereby the Icb1 $\Delta$ SLC1-1 cells are unable to tolerate heat, high salt and low $\mathrm{pH}$. The SLC1-1 mutation also rescues growth of certain $4 \Delta$ cells on $A b A$, although it is not known if its cell wall abnormality is alleviated (Vionnet et al., 2011). The C26-PI stereochemically resembles the normal IPCs, which also carry a C26 fatty acid on the C2-linked amino group of the LCB and recent data show that C26-PI needs to be mannosylated by Csg1p in order to suppress the growth inhibition imposed by myriocin (Vionnet et al., 2011). This points to the importance of MIPC in such sphingolipid depleted strains. Similarly, MIPC was also shown to be involved in recruiting the essential PI4P 5-kinase Mss4p to the plasma membrane and thereby activate Rom2p, a GEF of Rho1p and Rho2p, thus allowing for the activation of the cell wall integrity pathway, a process that enables cells to grow in the presence of myriocin (Kobayashi et al., 2005). Interestingly, recruitment of septins, in particular of Cdc10p to the bud neck and ordering them into filaments, may be dependent on $\mathrm{PI}$,5bisP also in yeast, in analogy to $S$. pombe, fruit flies and man, where PI4,5bisP has actually been shown to accumulate at the cleavage furrow (Bertin et al., 2010). However, it is not clear at present if the concentration of PI4,5bisP at the yeast bud neck is dependent on MIPCmediated Mss4p recruitment. Also, $\operatorname{csg} 1 \Delta$ and $\operatorname{csg} 2 \Delta$ (Fig. 1) were not picked up in screens testing the library of non-essential gene deletions for cell wall defects, suggesting that the Mss4p-recruitment by MIPC is absolutely required only in cells lacking the early sphingolipid biosynthetic intermediates.

Some membrane property is altered within $10 \mathrm{~min}$ of $\mathrm{AbA}$ treatment of yeast cells and triggers a regulatory feed back mechanisms that results in the activation of Lcb1p/ Lcb2p. The signal is transmitted through a TORC2-SIm1/ 2p-Ypk1p-Orm1/2p cascade (Roelants et al., 2011; Berchtold et al., 2012). As mechanical stress of the membrane also activates the Ypk1p-mediated Orm1/2p activation, it may be that it is a change in physical properties of the membrane rather than the absence of a specific sphingolipid that is sensed in this feedback loop. A good case has been made for MIPC to act as an activator of protein kinases Fpk1/2p, (Roelants et al., 2010). MIPC-activated Fpk1/2p and Ypk1p mutually phosphorylate and thereby inactivate each other, possibly allowing MIPC to downregulate the activation of $L c b 1 / 2 p$ that is caused by lack of complex sphingolipids (see above). In summary, some roles of MIPC may partly explain the cytokinesis defect of cSL $\Delta$ cells. The severity of this defect in $\operatorname{cSL} \Delta$, however, seems to suggest that complex sphingolipids may yet play a further, not-yet-known role in cell separation.

\section{Outlook}

The study of cSL $\Delta$, crippled as it is, may reveal new processes depending on complex sphingolipids. Similarly, use of GhLag1 will allow investigation in more detail of some cell biological processes that have been recognized to be dependent on complex sphingolipids and to ask specifically whether sphingolipids with very long chain fatty acids are required. GhLag1 also for the first time allow to acutely reduce IPC levels using $A b A$, remaining confident that the observed phenotypes are not the consequence of acute ceramide intoxication and imminent cell death. It is to be expected that many sphingolipid controlled stress situations will be studied in the GhLag1 background. 


\section{References}

Aguilar, P.S., Frohlich, F., Rehman, M., Shales, M., Ulitsky, I., Olivera-Couto, A., et al. (2010) A plasma-membrane E-MAP reveals links of the eisosome with sphingolipid metabolism and endosomal trafficking. Nat Struct Mol Biol 17: 901-908.

Barz, W.P., and Walter, P. (1999) Two endoplasmic reticulum (ER) membrane proteins that facilitate ER-to-Golgi transport of glycosylphosphatidylinositol-anchored proteins. $\mathrm{Mol}$ Biol Cell 10: 1043-1059.

Beeler, T., Bacikova, D., Gable, K., Hopkins, L., Johnson, C., Slife, H., and Dunn, T. (1998) The Saccharomyces cerevisiae TSC10/YBR265w gene encoding 3-ketosphinganine reductase is identified in a screen for temperature-sensitive suppressors of the Ca2+-sensitive csg2Delta mutant. J Biol Chem 273: 30688-30694.

Berchtold, D., Piccolis, M., Chiaruttini, N., Riezman, I., Riezman, H., Roux, A., et al. (2012) Plasma membrane stress induces relocalization of SIm proteins and activation of TORC2 to promote sphingolipid synthesis. Nat Cell Biol 14: 542-547.

Bertin, A., McMurray, M.A., Thai, L., Garcia, G., Votin, V., Grob, P., et al. (2010) Phosphatidylinositol-4,5bisphosphate promotes budding yeast septin filament assembly and organization. $J \mathrm{Mol}$ Biol 404: 711-731.

Cerantola, V., Vionnet, C., Aebischer, O.F., Jenny, T., Knudsen, J., and Conzelmann, A. (2007) Yeast sphingolipids do not need to contain very long chain fatty acids. Biochem J 401: 205-216.

Cerantola, V., Guillas, I., Roubaty, C., Vionnet, C., Uldry, D., Knudsen, J., and Conzelmann, A. (2009) Aureobasidin A arrests growth of yeast cells through both ceramide intoxication and deprivation of essential inositolphosphorylceramides. Mol Microbiol 71: 1523-1537.

Contreras, F.X., Ernst, A.M., Haberkant, P., Bjorkholm, P., Lindahl, E., Gonen, B., et al. (2012) Molecular recognition of a single sphingolipid species by a protein's transmembrane domain. Nature 481: 525-529.

Dickson, R.C. (2010) Roles for sphingolipids in Saccharomyces cerevisiae. Adv Exp Med Biol 688: 217-231.

Endo, M., Takesako, K., Kato, I., and Yamaguchi, H. (1997) Fungicidal action of aureobasidin A, a cyclic depsipeptide antifungal antibiotic, against Saccharomyces cerevisiae. Antimicrob Agents Chemother 41: 672-676.

Epstein, S., Castillon, G.A., Qin, Y., and Riezman, H. (2012) An essential function of sphingolipids in yeast cell division. Mol Microbiol 84: 1018-1032.

Fujita, M., Yoko-o, T., Okamoto, M., and Jigami, Y. (2004) GPI7 involved in glycosylphosphatidylinositol biosynthesis is essential for yeast cell separation. $J$ Biol Chem 279: 51869-51879.
Kobayashi, T., Takematsu, H., Yamaji, T., Hiramoto, S., and Kozutsumi, Y. (2005) Disturbance of sphingolipid biosynthesis abrogates the signaling of Mss4, phosphatidylinositol-4phosphate 5-kinase, in yeast. J Biol Chem 280: 1808718094.

Levine, T.P., Wiggins, C.A., and Munro, S. (2000) Inositol phosphorylceramide synthase is located in the Golgi apparatus of Saccharomyces cerevisiae. Mol Biol Cell 11: 2267-2281.

Nagiec, M.M., Wells, G.B., Lester, R.L., and Dickson, R.C. (1993) A suppressor gene that enables Saccharomyces cerevisiae to grow without making sphingolipids encodes a protein that resembles an Escherichia coli fatty acyltransferase. J Biol Chem 268: 22156-22163.

Nagiec, M.M., Nagiec, E.E., Baltisberger, J.A., Wells, G.B., Lester, R.L., and Dickson, R.C. (1997) Sphingolipid synthesis as a target for antifungal drugs. Complementation of the inositol phosphorylceramide synthase defect in a mutant strain of Saccharomyces cerevisiae by the AUR1 gene. J Biol Chem 272: 9809-9817.

Roelants, F.M., Baltz, A.G., Trott, A.E., Fereres, S., and Thorner, J. (2010) A protein kinase network regulates the function of aminophospholipid flippases. Proc Natl Acad Sci USA 107: 34-39.

Roelants, F.M., Breslow, D.K., Muir, A., Weissman, J.S., and Thorner, J. (2011) Protein kinase Ypk1 phosphorylates regulatory proteins Orm1 and Orm2 to control sphingolipid homeostasis in Saccharomyces cerevisiae. Proc Natl Acad Sci USA 108: 19222-19227.

Saba, J.D., Nara, F., Bielawska, A., Garrett, S., and Hannun, Y.A. (1997) The BST1 gene of Saccharomyces cerevisiae is the sphingosine-1-phosphate lyase. $J$ Biol Chem 272: 26087-26090.

Schorling, S., Vallee, B., Barz, W.P., Riezman, H., and Oesterhelt, D. (2001) Lag1p and Lac1p are essential for the Acyl-CoA-dependent ceramide synthase reaction in Saccharomyces cerevisiae. Mol Biol Cell 12: 3417-3427.

Tafesse, F.G., Huitema, K., Hermansson, M., van der Poel, S., van den Dikkenberg, J., Uphoff, A., et al. (2007) Both sphingomyelin synthases SMS1 and SMS2 are required for sphingomyelin homeostasis and growth in human HeLa cells. J Biol Chem 282: 17537-17547.

Tani, M., and Kuge, O. (2010) Defect of synthesis of very long-chain fatty acids confers resistance to growth inhibition by inositol phosphorylceramide synthase repression in yeast Saccharomyces cerevisiae. J Biochem 148: 565571.

Vionnet, C., Roubaty, C., Ejsing, C.S., Knudsen, J., and Conzelmann, A. (2011) Yeast cells lacking all known ceramide synthases continue to make complex sphingolipids and to incorporate ceramides into glycosylphosphatidylinositol (GPI) anchors. J Biol Chem 286: 6769-6779. 